\title{
Ontic structural realism as a metaphysics of objects
}

\author{
Michael Esfeld and Vincent Lam ${ }^{1}$ \\ University of Lausanne, Department of Philosophy, Michael-Andreas.Esfeld@unil.ch \\ University of Queensland, School of Mathematics and Physics \& \\ School of History, Philosophy, Religion and Classics, v.lam@uq.edu.au
}

(forthcoming in Alisa and Peter Bokulich (eds.): Scientific structuralism.

Dordrecht: Springer 2010. Chapter 8)

\begin{abstract}
The paper spells out five different accounts of the relationship between objects and relations three of which are versions of ontic structural realism (OSR). We argue that the distinction between objects and properties, including relations, is merely a conceptual one by contrast to an ontological one: properties, including relations, are modes, that is the concrete, particular ways in which objects exist. We then set out moderate OSR as the view according to which irreducible relations are central ways in which the fundamental physical objects exist. Physical structures thus consist in objects for whom it is essential that they are related in certain ways. There hence are objects, but they do not possess an intrinsic identity. This view can also admit intrinsic properties as ways in which objects exist provided that these do not amount to identity conditions for the objects. Finally, we indicate how this view can take objective modality into account.
\end{abstract}

\subsection{Introduction}

In a first approach, ontic structural realism (OSR) is a realism towards physical structures in the sense of networks of concrete physical relations, without these relations being dependent on fundamental physical objects that possess an intrinsic identity as their relata. In that vein, OSR has been developed in recent years as a metaphysics of contemporary fundamental physics, mainly non-relativistic quantum mechanics (QM), relativistic quantum field theory (QFT) and the general theory of relativity (GTR). The fundamental physical features of permutation invariance in many-particles quantum theory (Muller 2009), quantum entanglement in QM (Esfeld 2004) and in QFT (Lam 2010a), gauge invariance in quantum gauge theories (Lyre 2004) as well as background independence and gauge-theoretic diffeomorphism invariance in GTR (Rickles 2006, Esfeld and Lam 2008) have all been shown to support OSR in the following sense: these fundamental physical features can with good reason be taken to suggest all the same conclusion, namely that the fundamental physical objects - whatever they are according to the theory under consideration - are parts (relata) of a physical structure in the sense of a network of concrete physical relations. These objects do not have any existence - and in particular not any identity - independently of the structure they are part of (that is, the relations they bear to each other).

To be more precise, the original papers on OSR - mainly Ladyman (1998) and French and Ladyman (2003) - consider OSR to be supported by a fundamental underdetermination about

1 We are greatful to Tim Raez, Jakob Sprickerhof and Christian Wüthrich for helpful comments on the draft of this paper. 
individuality in QM and about quantum fields in QFT. Ladyman and Ross (2007, chapter 2 to 4), French (2010, sections 3-4) and French and Ladyman (2010 this volume, chapter 2.1) still defend such a view. Similarly, Bain $(2006,2009)$ argues in favour of an OSR interpretation of GTR on the basis of the different mathematical formulations of the theory. However, it is in dispute whether there really is such an underdetermination (see Cao 2003 and Pooley 2006, p. 90, against French and Ladyman 2003). We therefore take OSR to be directly and explicitly supported by the above mentioned features of QM and QFT as well as GTR.

These empirical arguments from contemporary fundamental physics stand against a long metaphysical tradition, which can be traced back to Aristotle. According to this tradition, physical objects, notably the fundamental ones, have to be something in themselves, enjoying an intrinsic identity. To be more precise, one can distinguish between the following three possible situations:

(a) The fundamental physical objects are equipped with an intrinsic identity: each fundamental physical object has intrinsic properties, that is, possesses properties that are independent of whether the object is alone or accompanied by other objects (see the definition of intrinsic properties by Langton and Lewis 1998). These properties are furthermore such that they distinguish each object from all the other objects that there are in the world. Aristotle sets out such a position, if one applies his considerations in Metaphysics Book VII to fundamental physical objects.

(b) The fundamental physical objects are not equipped with an intrinsic identity: they have (or at least can have) intrinsic properties, but no fundamental physical object has intrinsic properties that distinguish it from all the other fundamental physical objects. However, there are - asymmetric - relations that provide for identity conditions in that they distinguish each fundamental physical object from all the other ones. Time instants in Galilean space-time can serve to illustrate this situation: there are no intrinsic properties that distinguish each time instant from all the other ones. But the irreflexive and asymmetric relation "earlier than" does so.

(c) Neither intrinsic properties nor relations provide for identity conditions of the fundamental physical objects: two or more fundamental physical objects can have all the same intrinsic properties and stand in the same relations. This description has to be further qualified in view of what is known as weak discernibility in the current discussion; we will go into that issue at the end of the next section.

While situation (b) arguably is already a form of OSR, it is situation (c) that the mentioned features from contemporary fundamental physics on which OSR draws suggest. Whereas in situations (a) and (b), there are identity conditions that distinguish each object from all the other ones, provided for by either intrinsic properties (a) or relations (b), there are no identity conditions that distinguish objects from each other in situation (c). This situation therefore raises the question in what sense there are objects at all. Against this background, the main reservations about OSR are directed at the view of objects that this position is taken to imply. Accordingly, this paper aims to sketch out a metaphysics of objects within OSR, thus answering the question of the relationship between objects and relations.

We will first set out five different views of the relationship between objects and relations; three of them are versions of OSR (section 2). We will then introduce our own account that takes the distinction between objects and relations to be a conceptual rather than an ontological one, thus also amending our previous publications on OSR (notably Esfeld 2004 
and Esfeld and Lam 2008) (section 3). Finally, we briefly go into the issues of intrinsic properties (section 4) and objective modality (section 5).

\subsection{Ontological primacy and ontological dependence}

The issue of the relationship between objects and relations within OSR has mainly been addressed in the literature in terms of ontological primacy (Stachel 2006, Ladyman and Ross 2007, chapter 3.4, French 2010). We can distinguish five possibilities in order of increasing ontological importance of the relations over the objects (relata).

(1) There are only objects, but no non-supervenient relations. Objects have only intrinsic properties. All physical relations are reduced to the intrinsic properties of objects in the sense that they strongly supervene on them. Suppose that mass is an intrinsic property of objects. Relations such as being heavier than, being lighter than, having the same mass as then supervene on the masses that objects have each independently of one another. If one sets out to account for what there is in the world, it suffices to mention the intrinsic properties; the relations then come for free. The intrinsic properties furthermore equip the objects with an intrinsic identity: there is at least one intrinsic property of each object by means of which it is distinct from all the other objects in the world. Leibniz proposes such a radical atomistic conception in his Monadology (1714). Since there are no relations and thus no structures admitted in fundamental ontology, such a conception obviously does not count as a form of OSR.

This conception faces serious objections. At least spatio-temporal relations are generally admitted as being non-supervenient relations, even in classical physics. As is well known, Leibniz puts forward a good argument against spatio-temporal relations existing independently of matter in his correspondence with Newton-Clarke, but he by no means succeeds in showing spatio-temporal relations to supervene on intrinsic properties of objects (such as the monads).

(2) There are non-supervenient relations among objects - such as spatio-temporal relations in classical space-time prior to the theory of general relativity -, but there is an ontological primacy of the objects (relata) over the relations (and thus over the structures they are part of) in the following sense: the objects are equipped with an intrinsic identity independently of the relations in which they stand and thus independently of the other objects that happen to be in the world. Relations are irrelevant as far as the identity of the objects is concerned.

Admitting non-supervenient relations among objects whose identity does not depend on these relations is the standard way in traditional metaphysics to conceive the relationship between objects and relations. This view is not a form of OSR either, since it takes an intrinsic identity of the fundamental physical objects for granted. David Lewis' thesis of Humean supervenience can be regarded as the most famous example of such an atomistic position within contemporary metaphysics (e.g. Lewis 1986, introduction): Lewis recognizes spatio-temporal relations as being non-supervenient relations that hold the world together. However, these relations do not contribute to the identity of the fundamental material objects. That identity is constituted by fundamental physical, intrinsic properties occurring at spacetime points, the fundamental physical domain consisting in the distribution of intrinsic properties at space-time points. However, this conception is clearly at odds with quantum physics, since it is now commonly recognized that there are no intrinsic properties on which 
the relations of quantum entanglement could supervene (the same argument applies to QFT, even with a stronger force; see Lam 2010a).

(3) There are relations as well as objects standing in the relations without there being any ontological priority between them. Relations and objects are both genuine fundamental ontological entities. They are on the same ontological footing, being given "at once" in the sense that they are mutually ontologically dependent on each other. Note two consequences of this view:

- Since relations and objects are on the same ontological footing, it has to be accepted as a primitive that there is a numerical diversity of objects. Their numerical diversity is neither grounded in relations, nor is it grounded in intrinsic properties (since these do not constitute identity conditions for objects). Nonetheless, there is no question of haecceitism here (see Ladyman 2007, French and Ladyman 2010 this volume, chapter 2.3).

- Contrary to what Ainsworth (2010, pp. 53-54) claims, this conception does not deny that properties are part of fundamental ontology. Properties do not have to be intrinsic, but can also be relational (extrinsic) (see Hoffmann-Kolss 2010 for an elaborate study of extrinsic properties). If there are physical relations among objects as relata, these objects have relational properties, and the other way round.

This conception clearly is a variant of OSR. We have developed it under the name "moderate structural realism" in Esfeld (2004) and Esfeld and Lam (2008). This conception can accommodate both situation (b) and situation (c) described at the end of the preceding section, although it is (c) that is relevant in the context of current fundamental physics. Consequently, this conception has mainly been elaborated and advocated within the context of entanglement and non-separability in QM (Esfeld 2004) and with respect to GTR in order to account for the gauge-theoretic diffeomorphism invariance and the background independence postulated by that theory (Pooley 2006, Rickles 2006, Esfeld and Lam 2008; as regards moderate structural realism, see also Floridi 2008).

(4) Relations are ontologically primary and objects are ontologically secondary in the sense that they derive their existence from the relations in which they stand and thus from the structures they are part of. Objects are mere nodes within structures. The relations bear all the ontological weight: objects are literally constituted by the relations in which they stand. To the extent that this view recognizes intrinsic properties, it has to reconstruct them on the basis of relations as well. It can accommodate both situation (b) and situation (c) described at the end of the preceding section as well. Ladyman favours such a view on the basis of the mentioned physical features (see notably Ladyman and Ross 2007, chapter 2 to 4, and French and Ladyman 2010 this volume, chapter 2.3).

(5) There are only relations and no objects, thus no relata, in the domain of fundamental physics. There are only objectless structures in the sense of networks of relations without relata. Objects are not genuine fundamental ontological entities, it's relations (structures) all the way down. According to this radical conception, fundamental physics not only undermines the notion of intrinsic identity but the very notion of objects being part and parcel of fundamental ontology. This is the radical, eliminativist variant of OSR. French and Ladyman (2003) are commonly seen as setting out this version of OSR. It is still recently explicitly defended in French (2010, section 7). This position fits only situation (c) described at the end of the preceding section. There is no question of identity conditions for objects, since there simply are no objects in the last resort. 
French (2010, section 7) provides an alternative characterization of the OSR variants (3)(5) in terms of the ontological dependence between the objects and the structure to which they belong. Accordingly, he distinguishes three options for this ontological dependence: the case of a symmetric dependence, the case of an asymmetric dependence and the case where the dependence concerns the very essence of objects. Symmetric dependence characterizes (3), since if entities are mutually ontologically dependent on each other, none of them has an ontological priority over the others. Asymmetric dependence characterizes (4): objects derive their existence from and are constituted by the relations in which they stand. French's third option claims that it belongs to the very essence of an object that it exists only if the structure it is part of does. He elaborates on this option by maintaining that objects only exist "if the relevant structure exists and the dependence is such that there is nothing to them - intrinsic properties, identity, constitution, whatever - that is not cashed out, metaphysically speaking, in terms of this structure. What exists then are not objects in any ontological sense" (2010, p. 18). Hence, this option precisely characterizes (5). French (2010, section 7) furthermore argues that (3) and (4) entail such a thin notion of object that they in effect collapse into the eliminativist variant (5).

The alleged elimination of objects in fundamental ontology has provoked the major objection against OSR, namely that in repudiating objects, it is hardly intelligible as a metaphysical conception of the concrete physical world (Busch 2003, Cao 2003, Chakravartty 2003, Psillos 2006). Three aspects of this objection can be distinguished: (i) a metaphysical one according to which relations require some sort of relata as that what stands in the relations, even if these relata do not necessarily possess an intrinsic identity; (ii) an empirical one according to which the physical features on which OSR draws its support by no means suggest abandoning a commitment to there being objects in the fundamental physical domain (see notably Ainsworth 2010, p. 53); (iii) a logical one, concerning the quantification over objects in standard first order logic and the apparently unavoidable use of set-theoretical concepts within physical theories.

To counter (iii), Bain (2009) argues that the use of category-theoretic concepts in fundamental physics may provide the right framework for eliminativist OSR (5). Nonetheless, it is not clear to what extent these concepts are free from standard set-theoretic ones and what is the relevance of category theory for fundamental physics. We are therefore not convinced that we have any workable alternative at our disposal that is capable of avoiding the notion of objects or relata altogether and in what sense such an alternative would be preferable for the formulation and understanding of fundamental physical theories. As regards (i) and (ii), we do not wish to rule out on a priori reasons that a metaphysical position that rejects objects altogether may be consistent, and we clearly do not have any objection to a revisionary metaphysics based on science, being favourable to the methodological attitude advanced in Ladyman and Ross (2007, chapter 1). However, metaphysics should not be more revisionary than is required to account for the results of science, and in that respect, we do not see a cogent reason to abandon a commitment to objects.

As regards version (4) of OSR, this position sets out to reconstruct relata on the basis of relations, objects somehow emerging from relations as being nodes in a network of relations. However, the commitment to an ontological priority of relations over relata again invites the above mentioned objection in all its three aspects, for if objects somehow derive from 
relations, one still is committed to there being relations without relata in the fundamental physical domain in the first place. This objection hence applies not only to (5), but also to (4).

Nonetheless, it may seem that the idea of an ontological primacy of relations over relata, objects somehow deriving from relations, can draw support from recent claims about weak discernibility within QM. According to these claims, the identity of fundamental quantum physical objects - be they fermions, be they bosons - can always be grounded in the weak version of the principle of the identity of indiscernibles (Saunders 2006, Muller and Saunders 2008, Muller and Seevinck 2009). However, the physical significance of weak discernibility is in dispute, and we do not see how weak discernibility could ground an ontological priority of relations over relata.

The fact that two objects are weakly discernible is compatible with the two objects having all the same intrinsic properties and standing in the same relations, so that they cannot be distinguished. Weak discernibility merely means that there is a symmetric and irreflexive relation between the two objects. Nonetheless, the fact that the relation is irreflexive makes clear that there are two objects and not just one object, since nothing can stand in an irreflexive relation to itself. Consequently, weak discernibility tells us how many objects there are in the domain under consideration and thus gives us an epistemic, empirical access to these objects, but it does not distinguish these objects from each other. As Ladyman and Bigaj (2010) recently put it: “ . . the formal condition of weak discernibility, while being sufficient to establish numerical diversity, is not properly thought of as establishing that two objects can be discerned from each other. ... The relation that weakly discerns them does not amount to a difference between them over and above their numerical distinctness" (p. 130). In a similar vein, Dieks and Versteegh (2008) say: “... because of the symmetry any property or relation that can be attributed to one object can equally be attributed to any other and we can therefore not single out any specific object" (p. 926).

By way of consequence, weak discernibility does nothing to show how objects could be derived from relations, as claimed by position (4): it is not that the relations somehow constitute the relata and their plurality, these latter somehow deriving their existence from the relations, as intrinsic properties in traditional metaphysics may be taken to constitute the identity and plurality of objects by distinguishing each object from all the other ones (at least on a view that takes the identity of objects to be somehow constituted by their intrinsic properties instead of consisting in a primitive thisness or haecceity).

Nonetheless, there is a lesson to be learnt from weak discernibility in this context. As the quotation from Ladyman and Bigaj also makes clear, due to weak discernibility, there is no need to accept a numerical diversity of objects as a primitive, as in version (3) of OSR. Being committed to such a primitive numerical diversity is the main reservation against version (3). Due to weak discernibility, there is no primitive, naked numerical diversity of objects, being entities on a par with relations, there being a mutual ontological dependence between both of them. Insofar as there are objects, these stand in irreflexive, symmetric relations, although these relations do not distinguish them. We therefore take weak discernibility to support the position that we will set out in the next section. In case weak discernibility holds firm in the case of symmetric space-times such as the FLRW solutions of the Einstein field equations as well (Muller and Saunders 2008, p. 529, claim so), the reservations that Wüthrich (2009) voices in challenging the space-time structuralist could also be adressed. 
One may object against these considerations that the very fact to pose a domain of elements over which physical variables range presupposes acknowledging a numerical diversity of objects. However, Muller and Saunders (2008, pp. 542-544) convincingly argue that one should not mix formal, logical objects and their logical identity conditions with physical objects and their physical identity conditions, these latter being provided by physically meaningful predicates. As Muller (2009) puts it, "The numerical diversity is accounted for physically, that is, by means of physically meaningful and permutation-invariant relations" (p. 9).

We agree that the symmetric and irreflexive relations that amount to weak discernibility are physically meaningful. Consequently, we do not have to presuppose a numerical diversity of naked physical objects as a primitive in the metaphysics of fundamental physics. But we also wish to point out that the name "weak discernibility" is a misnomer, as Ladyman and Bigaj say (2010, p. 130), since the symmetric and irreflexive relations in question by no means amount to distinguishing one object from the other objects in the domain in question. As far as these relations are concerned, the objects are qualitatively, albeit not numerically, all the same. This simply is a lesson that current fundamental physics teaches us, admitting no stronger form of discernibility than the one that goes under the name of weak discernibility.

In sum, current fundamental physics does not make an intrinsic identity of the fundamental physical objects, whatever they may be, available. The relations or structures acknowledged in current fundamental physics cannot provide for an identity that distinguishes each object from the other ones either, since they yield no more than what is known as weak discernibility. However, weak discernibility does not contribute to vindicating the idea of relations enjoying ontological primacy over relata in that objects somehow emerge out of relations (4), and the other two versions of OSR - symmetric ontological dependence between objects and relations (3), eliminativism with respect to objects (5) - are not convincing either.

\subsection{A conceptual in contrast to an ontological distinction}

In order to overcome these metaphysical dead ends, we suggest that the distinction between objects and relations (or properties in general) is not to be regarded as an ontological one, as usually assumed, but only as a conceptual one, anchored in our thinking and language. The question of the ontological relationship between objects and relations is ill-posed. We predicate properties, including relations, of something, we quantify over objects, and we define a structure on a domain of objects by indicating how these objects are related to each other. However, this is the way in which we represent the world, its philosophical analysis going back to Aristotle's treatise On interpretation (Peri hermeneias). It does not match a real distinction in the world. Consequently, there is no point in enquiring into the relationship between objects and properties, including relations or structures, and, in particular, to talk in terms of a mutual ontological dependence between objects and properties, including relations or structures, or an ontological priority of the one over the others. There are not two types of entities, objects and properties including relations or structures, that entertain a certain relationship of ontological dependence. The dependence is only conceptual.

In recent philosophy of physics literature, Earman (2002) suggests a similar move in the interpretation of the GTR observables when he proposes that the subject-properties (or attributes) distinction belongs only to the representation of reality, but not to reality itself. French (2010, p. 18) also agrees that the distinction between objects and relations is only a 
conceptual one, but he does so against the background of an ontology that eliminates objects, namely radical OSR (5).

We can draw on Spinoza's metaphysics in order to make the idea more precise according to which objects and relations exist without the question of an ontological priority of the one over the others, or the question of a mutual ontological dependency, arising. Following Spinoza's Ethics (1677), properties are modes, that is, concrete, particular ways in which objects are. There is no ontological distinction between objects and their properties in the sense of modes: the modes are the way in which the objects exist. Objects do not have any existence in distinction to their ways of existence, and their ways of existence do not have any existence in distinction to the objects. One can draw a conceptual distinction between objects and their ways of existence, but not an ontological one, applying to reality. In reality, there is only one type of entity, namely objects that exist in particular ways. This theory of Spinoza is well worked out with respect to intrinsic properties, regarding the intrinsic properties as the ways in which the objects exist and providing for an identity of the objects that distinguishes each object from all the other ones in a given domain. This theory is taken up in contemporary metaphysics notably by Heil (2003, chapter 13) and Strawson (2008) (cf. also Armstrong 1989, pp. 96-98).

Nothing hinders to apply this theory to relations as well. Like intrinsic properties, relations can be the ways in which objects exist. In this case, the objects in a given domain do not exist independently of each other, being equipped with an intrinsic identity each. By contrast, insofar as there are identity conditions for the objects, these are provided for by the relations in which they stand. Thus, relations may make available identity conditions in the sense of distinguishing each object from all the other ones in a given domain (situation (b) described in section 1). Nonetheless, relations can be the ways in which objects exist without yielding identity conditions. For instance, relations of entanglement are the ways in which quantum objects exist, metrical relations are the ways in which space-time points exist, but these objects can stand all in the same relations. They are only weakly discernible, due to the relations being irreflexive, but symmetric.

Consequently, a structure consists in objects whose ways of existence are certain relations that they bear to each other. The objects hence do not exist apart from the structure they are part of. Qua ways in which the objects exist, the structures are networks of concrete physical relations, by contrast to abstract second order structures defined on objects and their intrinsic properties and by contrast to abstract mathematical structures (see Esfeld and Sachse 2010, chapter 2, in particular 2.5, for an elaboration of this view in the context of the metaphysics of properties).

As with any metaphysical position, there is a certain price to pay in order to make this position available. One has to abandon the view of properties being universals that are instantiated by particulars, that is, objects. In that framework, there indeed is an ontological distinction between properties qua universals and objects qua particulars that instantiate the universals. However, there are good reasons independently of OSR and the interpretation of fundamental physics to abandon that traditional metaphysical framework. Suffice it here to mention the fact that since the days of Plato and Aristotle, is has not been proven possible to render the notion of the instantiation relation that is central to this metaphysical framework intelligible. If one conceives properties qua universals as something that exists beyond the empirical world, the question remains unanswered what it means that the particulars, the 
objects, in the empirical world take part in or instantiate the universals. The problem that this question highlights has already been pointed out by Plato himself in the Parmenides (130e133a), and it still is an open issue. Conceiving the universals as abstract mathematical structures that are instantiated by concrete physical objects in the world does not help to make the relationship of instantiation intelligible. If one regards, following Aristotle, the universals as being inherent in the particulars (the objects) in the world, it remains an open question how numerically one and the same universal can exist in many different objects.

The main alternative in contemporary metaphysics to the view of properties as universals that are instantiated by particulars (objects) in the empirical world is to conceive properties as tropes. One may take the distinction between properties as tropes and modes in the sense of the ways in which objects exist to be only a verbal one. After all, "modus" is in this context the Latin translation of "tropos" in Ancient Greek. However, the trope theory of properties is often linked with the view of objects being bundles of tropes. If the tropes are relations instead of intrinsic properties, objects thus being conceived as bundles of relations, one gets at least close to version (4) of OSR and faces the objections that this view provokes.

The position according to which relations are the ways (modes) in which objects exist clearly is a version of OSR. We propose to call this position "moderate ontic structural realism", thus amending our earlier view in Esfeld (2004) and Esfeld and Lam (2008) in which we defined moderate structural realism in terms of a mutual ontological dependence between objects and relations. This position is a moderate in contrast to a radical form of OSR (such as (5) and, less radical, (4)), because it recognizes objects. If relations are the ways (modes) in which objects exist, there is a clear and meaningful sense in which there are fundamental physical objects in the world, thus avoiding the mentioned objections against the versions (4) and (5) of OSR. These are not some sort of Ersatz objects, but objects tout court, as conceived in one of the main metaphysical positions on objects, namely the one associated with Spinoza according to which properties are the ways (modes) in which objects exist. Maintaining that intrinsic properties or relations are the ways in which objects exist is one thing, the question of whether or not intrinsic properties or relations are capable of providing for identity conditions of objects in that they are able to distinguish each object from all the other ones in a given domain is another matter. In other words, the question whether the relationship between objects and properties is only a conceptual or also an ontological affair and the question of identity conditions for objects are two separate issues.

By shifting from the mainstream metaphysical view of the modes being intrinsic properties to the view of the modes being relations, there is indeed no question of an intrinsic identity of objects, given by certain intrinsic ways of their existence. It is not required for an entity to be an object that it possesses an intrinsic identity or that it be distinct from all the other objects that there are in the world (see also Brading 2010 this volume, chapter 3.4). If one wishes to call objects that do not possess an intrinsic identity, following French and Ladyman (2010 this volume, chapter 2), thin objects, we do not have any objection against that manner of talking. Objects, as conceived by moderate ontic structural realism, still are genuine objects, but they are remarkably distinct from what traditional metaphysics takes objects to be, namely possessing an intrinsic identity. We prefer to make this issue clear by maintaining that the objects that moderate ontic structural realism admits are not individuals - since there is nothing, not even relations, that distinguishes each object in a given domain from all the other ones -, but one may also talk in terms of thin objects. 
Moderate structural realism, thus conceived, has to accept a numerical plurality of objects being related in certain ways as primitive - such as a numerical plurality of quantum objects being related by entanglement, or a numerical plurality of space-time points being related by metrical field properties. Note however that this numerical diversity is not one of naked objects, being ontologically distinct from properties including relations, but a diversity of objects related in certain manners and being weakly discernible due to the relations being irreflexive and symmetric. The relations thus tell us how many objects there are.

Any metaphysical position has to accept something as primitive. The numerical plurality of objects being related in certain ways is a primitive on a par with the plurality of fundamental intrinsic properties occurring at space-time points that Lewis accepts as primitive in his thesis of Humean supervenience, the plurality of instantiations of properties qua universals that the theory of properties as universals accepts as primitive, the plurality of tropes that may perfectly resemble each other which trope ontology endorses as primitive, etc. Insofar as these primitives are unobjectionable in the respective metaphysical framework, so is the numerical plurality of objects being related in certain ways in the framework of moderate ontic structural realism against the background of the fact that current physics does not yield more than what is known as weak discernibility for the fundamental physical objects.

Furthermore, this position is able to take into account a view in the philosophy of fundamental physics that may be called super-holism. It is sometimes suggested that in an EPR-Bell-experiment and thus in the singlet state, there are prior to measurement not two quantum objects that are related by entanglement (as proposed by Teller 1986 in his seminal paper on relational holism), but only one total object that has the disposition to manifest itself in measurement as two objects whose observables are correlated in a certain way (see e.g. Morganti 2009, p. 1031, Ainsworth 2010, p. 54, and the reservations that Dieks and Versteegh 2008, pp. 928-934, voice against speaking of two weakly discernible objects in a case like the singlet state). Since entanglement is generic in quantum theory, this view amounts in the end to the position that as regards fundamental ontology, there is only one object that has the disposition to manifest itself in the form of quantum objects that are correlated in certain ways. According to the position Spinoza sets out in his Ethics (1677), there is only numerically one substance, that is, one object, which Spinoza identifies with God; all the properties in the world are ways (modes) in which that one substance exists. If there is only one object in the last resort, there is of course no longer a question of physical relations being fundamental. OSR would thus not be the most fundamental ontology. There would rather be only one object that has the disposition to give rise to structures in the sense of a plurality of objects that are correlated in certain ways.

However, we remain sceptical with respect to such a super-holistic position, at least for the time being. It is entirely unclear how the one object could lead to structures in the mentioned sense as something that emerges out of it. The field interpretation of Spinoza put forward by Bennett (1984) is of no help in this context, since it conceives Spinoza's one object as classical, three-dimensional space. In the context of applying this super-holism to quantum theory, by contrast, a classical space or space-time - or a field defined on classical space-time - is obviously not a suitable candidate for the one object. Taking GTR into account, spacetime cannot even be presupposed as a background. Nonetheless, given the current research programmes in quantum gravity that envisage reconstructing space-time as somehow emerging out of a fundamental quantum domain, we do not wish do exclude that such a 
super-holism may in the future become a serious contender in the philosophy of physics. Our point here merely is that if this should turn out to be the case - and OSR hence not hold firm as a candidate for a most fundamental ontology - the view of properties, whatever they may be in concrete physical terms, being modes, that is, the ways in which an object exists, will presumably still stand. In a nutshell, this view is fruitful for OSR in that it dispels the ontological worries about the relationship between objects and relations (structures), and it would still remain valid even if it turned out that the structures can be traced back to ways of existence (modes) of numerically one object, the only object that there is.

\subsection{OSR and intrinsic properties}

Fundamental physical intrinsic (state-independent) properties, such as notably rest mass and the various quantum charges within quantum theory as well as total spin, constitute a notorious difficulty for OSR (for a recent version of this objection against OSR, see Ainsworth 2010, p. 54). If these properties are understood as genuine intrinsic properties - as they usually are, although they do not provide for identity conditions for quantum objects -, then it seems that none of the above three versions (3)-(5) of OSR can take them into account. Given that these are fundamental physical properties, this fact would constitute an important objection to OSR.

Of course, proponents of OSR in any of the three versions (3)-(5) have elaborated a defence. The main idea is to consider these properties as being defined in terms of symmetry relations (Castellani 1998): mass and spin can be defined as eigenvalues of the Casmir operators of the relevant symmetry group (the Galilei group in the non-relativistic case, the Poincaré group in the relativistic case), that is, as invariants of this group; similarly, quantum charges are defined in terms of the generators of the relevant quantum gauge groups (for instance, U(1) for the electric charge). Fundamental state-independent properties of quantum objects can therefore be understood in terms of symmetry relations. In that sense, OSR (in any of the above three versions) can readily account for them (Muller 2009, § 4).

However, it is questionable to what extent such an account is satisfactory. There are two main reservations: first, in order to conceive the fundamental physical state-independent properties in terms of physical relations, the symmetry relations have to be regarded as concrete physical relations, being in that respect on a par with the concrete physical relations of entanglement in which the state-dependent properties of quantum systems are implicated. It is debatable to say the least whether such an understanding of quantum gauge symmetry relations is a serious option. Second, even within this group-theoretic understanding of the fundamental state-independent properties, it seems that these latter can still be considered as intrinsic in the standard sense of being independent of accompaniment or loneliness. This second difficulty could possibly be avoided if one takes into account that strictly speaking the definition of state-independent properties requires some fixed space-time background structures (this is especially true for mass-energy within the dynamical space-times of GTR; see Lam 2010b).

Refusing to recognize the distinction between objects and relations (and properties in general) as an ontological one puts us in the position to avoid these troubles about stateindependent properties. Against this background, the OSR proponent can coherently accept them as candidates for genuine intrinsic properties without affecting the central claim of OSR: even if some of the ways fundamental physical objects are consist in intrinsic properties, 
important features of fundamental physics show that there always are central ways in which these objects exist that consist in relations, so that the objects do not have any existence - and in particular not any identity - independently of the structure they are part of. This is the third point in which we wish to amend the position set out as moderate ontic structural realism in Esfeld (2004) and Esfeld and Lam (2008): we no longer take OSR - at least in the moderate version that we defend, recognizing objects - to be opposed to acknowledging the existence of intrinsic properties, as long as such intrinsic properties do not amount to an intrinsic identity of the objects. It may be that there are intrinsic properties, or it may finally turn out that there are only relations in the fundamental physical domain. We wish to leave this question open. It is of minor importance for OSR as we propose to conceive this position.

\subsection{OSR and modality}

The structures to which OSR is committed are usually conceived as being modal structures (French and Ladyman 2003, Ladyman and Ross 2007, chapters 2 to 4, Esfeld 2009, French 2010, section 3, French and Ladyman 2010 this volume, chapter 2.1; but see Sparber 2009, chapter 4, and Lyre 2010 for Humean versions of OSR that reject objective modalities). OSR is opposed to a position that accepts physical laws as something primitive (Maudlin 2007 can be read as endorsing such a position). If structures are the fundamental ontological ingredient, then the laws supervene on the structures. That is to say, what the laws of nature are depends on what are the concrete physical structures that exist in the world. However, taking the laws to supervene on the structures does not imply a commitment to objective modality, regarding the structures themselves to be modal in some sense, for such a position is also available to a Humean. According to Lewis' thesis of Humean supervenience, the laws of nature that hold in a world supervene on the distribution of the fundamental intrinsic properties that happens to exist in the world in question, the laws being, in short, the salient regularities that this distribution exhibits (see e.g. Lewis 1994). Such a position is also available to a proponent of OSR, replacing the distribution of the fundamental physical intrinsic properties with the distribution of the fundamental physical structures (Sparber 2009, chapter 4.3.1). Hence, the mere fact of the laws supervening on the structures according to OSR does not amount to a commitment to the structures being modal.

One sense in which the structures can be modal is by being causal. One thus is committed to a realism about causation that takes causation to be a feature of the fundamental physical domain. Indeed, not being able to account for causation is one of the stock objections against OSR (e.g. Psillos 2006). There are three ways of replying to this objection: (a) One can maintain in the tradition of Russell's (1912) attack on causation that this objection is misplaced, since there is no causation in the domain of fundamental physics (Ladyman 2008). (b) One can take this objection at face value and admit causal properties that underlie the structures, thus abandoning OSR (Chakravartty 2007, chapters 3 to 5, can be read as making such a move). (c) One can regard the structures as being causal in themselves (Esfeld 2009; see furthermore Esfeld and Sachse 2010, chapter 2, for metaphysical and physical arguments in favour of objective modality and causal realism in the domain of fundamental physics).

Taking the structures to be causal is to say that insofar as there are concrete physical relations as the ways in which the fundamental physical objects exist, these are powers or dispositions to bring about certain physical effects (or, in other words, these bestow on the objects whose ways of existence they are the power to bring taken together certain effects 
about). To indicate an example of what this idea is to say in concrete terms, within the framework of the GRW interpretation of QM, one can maintain that entangled states are the power or disposition to bring about classical physical properties that have a rather precise value through spontaneous localizations (see Dorato and Esfeld 2010 for details). Since the entangled states are non-separable and thus not states that are localized at a certain point in physical space-time, the idea that quantum objects standing in entangled states have, insofar as the relations of entanglement are their way of existence, taken together the power or disposition to bring about rather well localized, classical physical properties through spontaneous localization does not pose any additional problems to making a collapse interpretation of QM such as GRW compatible with special relativistic physics (but does nothing to solve these problems either; see Tumulka 2006 for an important step towards achieving a relativistic version of GRW).

However, if in future research on quantum gravity, the quantum structures of entanglement should indeed turn out to be more fundamental than classical space-time, these problems may find a solution. Suffice it here to mention the fact that the research programmes that take space-time to emerge out of a more fundamental quantum domain are compatible with considering this emergence in causal terms. It may thus turn out to be possible to regard the fundamental quantum structures as the power or disposition to bring about space-time. In brief, it is conceptually possible to conceive the fundamental physical structures as modal structures qua being causal structures, and such a view is not only supported by certain interpretations of QM, but may also prove to be fruitful for a future metaphysics of quantum gravity.

In conclusion, we have set out in this paper a revised version of the position introduced as moderate ontic structural realism in Esfeld (2004) and Esfeld and Lam (2008) by maintaining that the distinction between objects and properties, including relations and thus structures, is only a conceptual one by contrast to an ontological one: properties, including relations, are modes, that is, the ways in which objects exist. Moderate OSR maintains that at least some central ways in which the fundamental physical objects exist are relations so that these objects do not have any existence - and in particular not any identity - independently of the structure they are part of. There thus clearly are fundamental physical objects, moderate OSR being a metaphysics of objects, but there is no question of a mutual ontological dependence between objects and relations, no question of having to accept a naked numerical diversity of objects as primitive, and this position also enables us to recognize genuine intrinsic properties as ways in which the fundamental physical objects exist without jeopardizing OSR. It can furthermore accommodate a modal nature of structures in conceiving them as causal powers.

\section{References}

Ainsworth, Peter M. (2010): "What is ontic structural realism?" Studies in History and Philosophy of Modern Physics 41, pp. 50-57.

Armstrong, David M. (1989): Universals. An opinionated introduction. Boulder (Colorado): Westview Press.

Bain, Jonathan (2006): “Spacetime structuralism”. In: D. Dieks (ed.): The ontology of spacetime. Amsterdam: Elsevier. Pp. 37-66.

Bain, Jonathan (2009): "Motivating structural realist interpretations of spacetime". Manuscript.

Bennett, Jonathan (1984): A study of Spinoza's “Ethics”. Indianapolis: Hackett.

Brading, Katherine (2010): "Structuralist approaches to physics: objects, models and modality". This volume, chapter 3 . 
Busch, Jacob (2003): "What structures could not be". International Studies in the Philosophy of Science 17, pp. 211-223.

Cao, Tian Yu (2003): "Can we dissolve physical entities into mathematical structure?" Synthese 136, pp. 57-71.

Castellani, Elena (1998): "Galilean particles: an example of constitution of objects". In: E. Castellani (ed.): Interpreting bodies: classical and quantum objects in modern physics. Princeton: Princeton University Press. Pp. 181-194.

Chakravartty, Anjan (2003): “The structuralist conception of objects". Philosophy of Science 70, pp. 867-878.

Chakravartty, Anjan (2007): A metaphysics for scientific realism: knowing the unobservable. Cambridge: Cambridge University Press.

Dieks, Dennis and Versteegh, Marijn A. M. (2008): "Identical quantum particles and weak discernibility". Foundations of Physics 38, pp. 923-934.

Dorato, Mauro \& Esfeld, Michael (2010): "GRW as an ontology of dispositions". Studies in History and Philosophy of Modern Physics 41, pp. 41-49.

Earman, John (2002): "Thoroughly modern McTaggart: or, what McTaggart would have said if he had read the general theory of relativity". Philosophers Imprint 2 (3).

Esfeld, Michael (2004): "Quantum entanglement and a metaphysics of relations". Studies in History and Philosophy of Modern Physics 35, pp. 601-617.

Esfeld, Michael (2009): "The modal nature of structures in ontic structural realism". International Studies in the Philosophy of Science 23, pp. 179-194.

Esfeld, Michael \& Lam, Vincent (2008): “Moderate structural realism about space-time”. Synthese 160, pp. 2746.

Esfeld, Michael \& Sachse, Christian (2010): Kausale Strukturen. Einheit und Vielfalt in der Natur und den Naturwissenschaften. Berlin: Suhrkamp. English translation Conservative reductionism forthcoming London: Routledge 2011.

Floridi, Luciano (2008): “A defence of informational structural realism”. Synthese 161, pp. 219-253.

French, Steven (2010): "The interdependence of structure, objects and dependence". Forthcoming in Synthese.

French, Steven \& Ladyman, James (2003): "Remodelling structural realism: quantum physics and the metaphysics of structure". Synthese 136, pp. 31-56.

French, Steven \& Ladyman, James (2010): "In defence of ontic structural realism". This volume, chapter 2.

Heil, John (2003): From an ontological point of view. Oxford: Oxford University Press.

Hoffmann-Kolss, Vera (2010): The metaphysics of extrinsic properties. Frankfurt (Main): Ontos.

Ladyman, James (1998): "What is structural realism?" Studies in History and Philosophy of Modern Science 29, pp. 409-424.

Ladyman, James (2007): “On the identity and diversity of objects in a structure". Proceedings of the Aristotelian Society. Supplementary Volume 81, pp. 1-22.

Ladyman, James (2008): "Structural realism and the relationship between the special sciences and physics". Philosophy of Science 75, pp. 744-755.

Ladyman, James \& Bigaj, Tomasz F. (2010): "The principle of the identity of indiscernibles and quantum mechanics”. Philosophy of Science 77, pp. 117-136.

Ladyman, James \& Ross, Don with Spurrett, David \& Collier, John (2007): Every thing must go. Metaphysics naturalised. Oxford: Oxford University Press.

Lam, Vincent (2010a): “The non-intrinsic identity of fundamental quantum objects”. Manuscript, submitted.

Lam, Vincent (2010b): "Gravitational and non-gravitational energy: the need for background structures". Forthcoming in Philosophy of Science.

Langton, Rae \& Lewis, David (1998): "Defining 'intrinsic". Philosophy and Phenomenological Research 58, pp. 333-345.

Lewis, David (1986): Philosophical papers. Volume 2. Oxford: Oxford University Press.

Lewis, David (1994): "Humean supervenience debugged". Mind 103, pp. 473-490. 
Lyre, Holger (2004): "Holism and structuralism in U(1) gauge theory". Studies in History and Philosophy of Modern Physics 35, pp. 643-670.

Lyre, Holger (2010): "Humean perspectives on structural realism". Proceedings of the 1st plenary conference of the ESF programme Philosophy of science in a European perspective. Berlin: Springer.

Maudlin, Tim (2007): The metaphysics within physics. Oxford: Oxford University Press.

Morganti, Matteo (2009): "A new look at relational holism in quantum mechanics". Philosophy of Science 76, pp. 1027-1038.

Muller, Fred A. (2009): "Withering away, weakly". Forthcoming in Synthese.

Muller, Fred A. \& Saunders, Simon (2008): "Discerning fermions". British Journal for the Philosophy of Science 59, pp. 499-548.

Muller, Fred A. \& Seevinck, Michiel (2009): "Discerning elementary particles". Philosophy of Science 76, pp. $179-200$.

Pooley, Oliver (2006): "Points, particles, and structural realism". In: D. Rickles, S. French \& J. Saatsi (eds.): The structural foundations of quantum gravity. Oxford: Oxford University Press. Pp. 83-120.

Psillos, Stathis (2006): "The structure, the whole structure and nothing but the structure". Philosophy of Science. Proceedings 73, pp. 560-570.

Rickles, Dean (2006): “Time and structure in canonical gravity”. In: D. Rickles, S. French \& J. Saatsi (eds.): The structural foundations of quantum gravity. Oxford: Oxford University Press. Pp. 152-195.

Russell, Bertrand (1912): "On the notion of cause". Proceedings of the Aristotelian Society 13, pp. 1-26.

Saunders, Simon (2006): “Are quantum particles objects?” Analysis 66, pp. 52-63.

Sparber, Georg (2009): Unorthodox Humeanism. Frankfurt (Main): Ontos.

Stachel, John (2006): "Structure, individuality, and quantum gravity". In: D. Rickles, S. French \& J. Saatsi (eds.): The structural foundations of quantum gravity. Oxford: Oxford University Press. Pp. 53-82.

Strawson, Galen (2008): "The identity of the categorical and the dispositional". Analysis 68, pp. 271-282.

Teller, Paul (1986): "Relational holism and quantum mechanics". British Journal for the Philosophy of Science 37, pp. 71-81

Tumulka, Roderich (2006): “A relativistic version of the Ghirardi-Rimini-Weber model”. Journal of Statistical Physics 125, pp. 821-840.

Wüthrich, Christian (2009): “Challenging the spacetime structuralist". Philosophy of Science 76, pp. 1039-1051. 